REVISTA CIENCIAS BIOMÉDICAS

PRESENTACIÓN DE CASOS CLÍNICOS

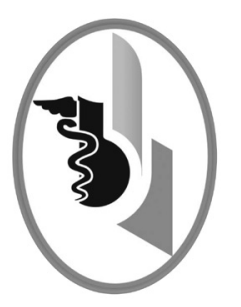

\title{
A PROPÓSITO DE UN CASO DE AMILOIDOSIS NODULAR CUTÁNEA
}

\author{
REGARDING A CASE OF NODULAR CUTANEOUS \\ AMYLOIDOSIS
}

Olaya-Villarreal Guillermo ${ }^{1}$

Mendivil-Silva César ${ }^{2}$

Pretelt-Mendoza José ${ }^{3}$

Redondo-Bermúdez César ${ }^{4}$

Correspondencia: guillemedicine@gmail.com

Recibido para evaluación: octubre - 20 - 2015. Aceptado para publicación: marzo - 15 - 2016.

\section{RESUMEN}

Introducción: la amiloidosis cutánea primaria de tipo nodular es un subtipo de amiloidosis de presentación exclusivamente cutánea que afecta principalmente a mujeres, se presenta con nódulos amarillentos brillantes y firmes al tacto, generalmente asintomáticos.

Caso clínico: se presentó el caso de una paciente de género femenino de 46 años con sintomatología de cuatro años de evolución consistente en la aparición de lesiones papulosas pequeñas en miembros inferiores y en la región lumbosacra, pruriginosas, que no respondieron al tratamiento inicial por medicina general con loratadina. Sin antecedentes personales de importancia. Se sospecha la presencia de amiloidosis; la histología con tinción Rojo Congo confirmó el diagnóstico. No se encontraron hallazgos de enfermedad sistémica.

Conclusión: la amiloidosis nodular, generalmente, es una enfermedad de etiología diversa, inflamatoria, crónica, poco frecuente y de afectación local en la piel con un pronóstico bueno. En ocasiones su tratamiento se ve retardado por el diagnóstico tardío. Rev.cienc.biomed. 2016;7(1):123-127.

\section{PALABRAS CLAVE}

Amiloidosis; Dermatosis de la pierna; Integumento común.

\section{SUMMARY}

Introduction: nodular primary cutaneous amyloidosis is a subtype of amyloidosis that is only cutaneous it mainly affects women. It comes up with yellow and bright nodules, hard to the touch, usually asymptomatic.

Case report: the case is presented in a 62-year-old female patient, who during four years presented pruritus, little papular lesions in lower limbs and in lumbosacral re-

Médico. Estudiante de Postgrado. Medicina Interna. Facultad de Medicina. Universidad de Cartagena. Cartagena. Colombia.

Médico. Estudiante de Postgrado. Patología. Facultad de Medicina. Universidad de Cartagena. Cartagena. Colombia.

Médico. Especialista en Dermatología. Universidad Nacional Autónoma de México. México.

Médico. Especialista en Patología. Docente Departamento Diagnóstico. Facultad de Medicina. Universidad de Cartagena. Cartagena. Colombia. 
gion that did not answered to the first medical treatment with loratadine. The patient did not present important clinical record. It is believed the presence of amyloidosis; the histology with Congo red staining confirmed the diagnosis. Systemic disease was not found.

Conclusion: usually, nodular amyloidosis is a disease with chronic, inflammatory, diverse and uncommon etiology; it only affects the skin with good prognosis. Sometimes, its treatment is delayed by late diagnosis. Rev.cienc.biomed. 2016;7(1):123-127.

\section{KEYWORDS}

Amyloidosis; Leg dermatoses; Integumentary system.

\section{INTRODUCCIÓN}

La enfermedad por depósitos de material amiloide es una patología crónica, sistémica o localizada, cuya forma sistémica puede corresponder a amiloidosis primaria o secundaria, esta última se denomina adquirida. La amiloidosis cutánea es localizada, de ella existen tres tipos: amiloidosis liquénica o papular, amiloidosis macular y amiloidosis nodular (1).

Rudolf Virchow fue el primero en introducir el término de amiloidosis, grupo de enfermedades caracterizadas por el depósito extracelular de una sustancia fibrilar de origen proteico, insoluble (amiloide) en distintos tejidos, con pérdida de la estructura normal del tejido, lo que lleva a disfunción en mayor o menor grado según la cuantía del depósito en una amplia variedad de situaciones clínicas (2-4).

\section{CASO CLÍNICO}

Paciente femenina de 46 años de edad, residente y procedente de Cartagena Colombia, quien consultó por cuadro clínico de dos meses de evolución, consistente en aparición de lesiones papulosas en miembros inferiores y región lumbosacra. La paciente negó antecedente de enfermedades sistémicas, consumo de alcohol o cigarrillo, consumo de medicamentos o antecedentes familiares de enfermedades hereditarias.

En el examen físico se observó dermatosis diseminada en el tronco que afectó especialmente el área dorsolumbar. También se encontraron similares lesiones en las piernas de forma bilateral y simétrica. Las lesiones estaban constituidas por placas de múltiples lesiones micropapulares brillantes, con eritema y fondo de color anaranjado (Figuras $\mathrm{N}^{\circ} 1$ y $\mathrm{N}^{\circ} 2$ ).

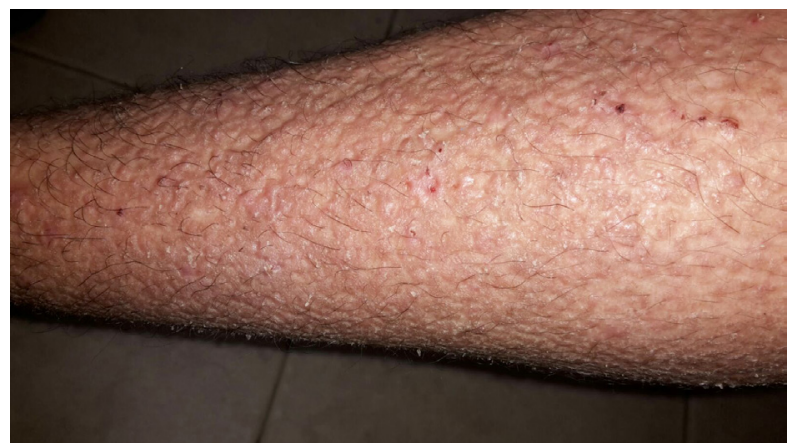

Figura No 1.

Lesiones en cara anterior de pierna.

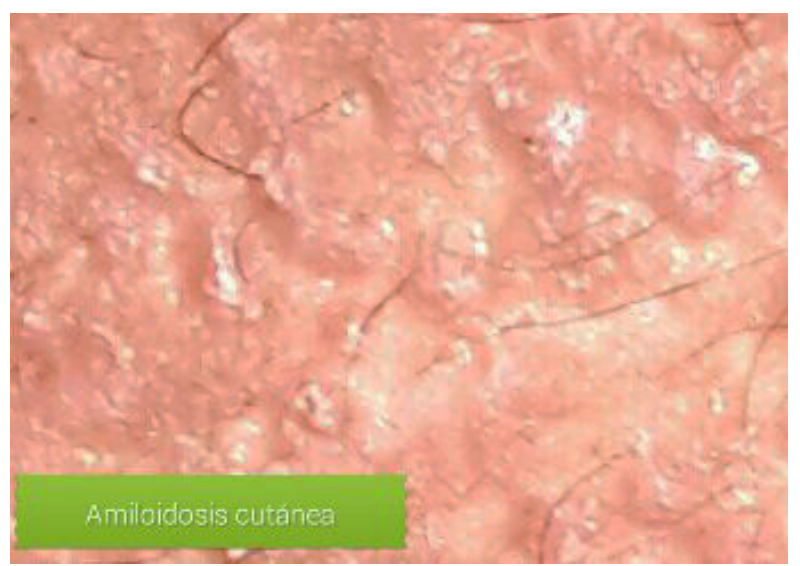

Figura No 2.

Lesión papular brillante.

Se realizaron los siguientes estudios: GOT: 22 u/lt. GPT: 19 u/lt; albuminuria: negativa; creatinina en orina: $124.4 \mathrm{mg} / 24$ horas; relación albúmina/creatinina: 5; parcial de orina: sin alteraciones; hemograma: leucocitos 7970, neutrófilos 72.4\%, linfocitos 22.4\%; HB: $11.7 \mathrm{~g} / \mathrm{dl}$, plaquetas: $307.000 \times \mathrm{mm}^{3}$; glicemia: $82 \mathrm{mg} / \mathrm{dl}$. En el electrocardiograma se detectó frecuencia 
cardiaca de 89 latidos por minuto con ritmo sinusal, sin isquemia aguda; eje normal; QT $445 \mathrm{~m} / \mathrm{seg;}$ la ecografía abdominal mostró hígado graso esteatósico grado-I; radiografía de tórax: mínima fibrosis en bases pulmonares y radiografía de cráneo sin hallazgos patológicos.

Serealizó biopsia de piel. En los cortes se observó epidermis con hiperplasia, hiperqueratosis compacta, hipergranulosis focal, acantosis irregular y algunos queratinocitos apoptóticos. En la dermis se observó depósito material rosado, amorfo, especialmente en la dermis reticular (Figura No 3 y No 4). La coloración Rojo Congo fue positiva para amiloide.

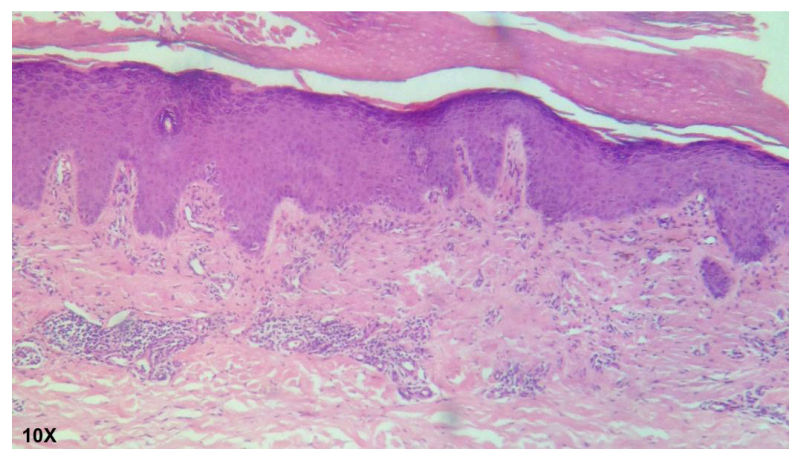

Figura No 3.

Hiperqueratosis ortoqueratósica e infiltrado mononuclear perivascular superficial.

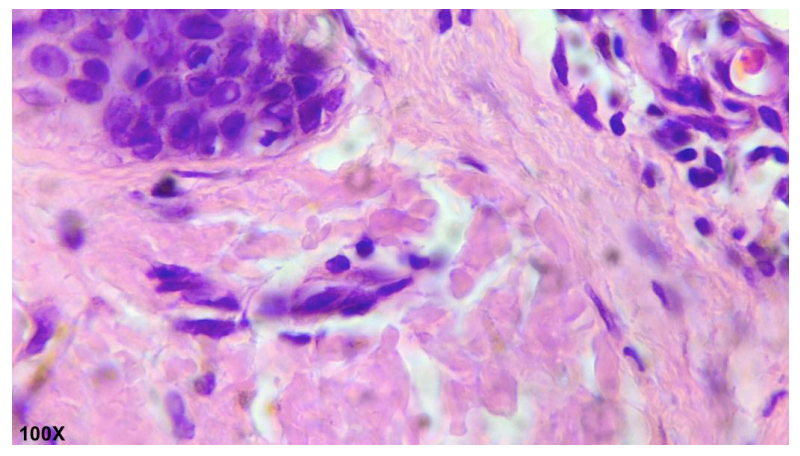

Figura No 4.

Depósitos de proteína amiloide.

\section{DISCUSIÓN}

La enfermedad por depósito de amiloide engloba un grupo de entidades sistémicas o localizadas con una característica común: la acumulación y depósito de esta sustancia proteica. La amiloidosis cutánea se diferencia de la amiloidosis sistémica en que el depósito se localiza exclusivamente en la piel, sin compromiso de los órganos internos (1).
Es una enfermedad infrecuente, con promedio anual de ocho casos por cada millón de habitantes. Es más frecuente después de los 40 años. Rudolf Virchow fue el primero en introducir el término de amiloidosis para denominar el grupo de enfermedades caracterizadas por el depósito extracelular de una sustancia fibrilar de origen proteico e insoluble. Por otra parte, esta enfermedad conlleva a la pérdida de la estructura normal del tejido, lo que genera disfunción en mayor o menor grado según la cuantía del depósito (2).

Inicialmente, el proceso fue llamado degeneración lardácea, que deriva del latín laridum, semejante a la manteca, posteriormente, al conservar dichos órganos en yodo, se comprobó que adquirían coloración azul semejante al almidón. Fue así como lo llamaron amiloidosis que viene del griego amylom, que significa almidón, y eidos forma (2).

Se distinguen dentro de la anatomía patológica dos formas dependiendo del tipo de microscopía usada. Con la microscopía óptica los depósitos de amiloide se tiñen de color rosa con hematoxilina-eosina. Con la tinción de Rojo Congo presentan birrefringencia verde manzana, muy característica cuando se aplica sobre cortes tisulares y se visualiza bajo luz polarizada. También se puede usar Tioflavina $\mathrm{T}$, que produce una intensa fluorescencia amarillo verdosa, otra forma es con azul alcián que presenta tinción verde. En la microscopía electrónica el amiloide está compuesto por una red de fibrillas no ramificadas de 75 a $100 A^{\circ}$ unidas entre sí. Todas las formas de amiloidosis son Rojo Congo positivas, y lo que determina el tipo de amiloidosis es el estudio inmunohistoquímico (1-3).

Clínicamente las máculas son manifestaciones frecuentes que consisten en manchas marrones oscuras o grises de dos a tres milímetros de diámetro, que se caracterizan por ser pruriginosas, generalmente localizadas en el tórax, extremidades y cara. Cuando la amiloidosis se asocia a mieloma múltiple y a otras gammapatías monoclonales, se observan en la piel y en las mucosas manifestaciones hemorragíparas. Ello ocurre principalmente en el párpado superior de los ojos, signo denominado "ojos de mapache", que se 
instaura especialmente luego de maniobra de Valsalva, esfuerzo, etc. En esas lesiones se encuentran depósitos de amiloide y cadenas ligeras de inmunoglobulinas, parece que el amiloide se produce localmente por las células plasmáticas del infiltrado y no en las de la médula ósea. Los depósitos de amiloide son indistinguibles de los observados en las amiloidosis sistémicas asociadas a discrasia de células plasmáticas (4).

Las lesiones de piel se presentan como un nódulo 0 varios nódulos subcutáneos eritematosos amarronados, de aspecto céreo y consistencia firme. El número y el tamaño son variables, generalmente entre uno y tres centímetros. Con frecuencia presentan telangiectasias y hemorragia (color púrpura) en la superficie. A veces en el centro se aprecia atrofia; las lesiones son asintomáticas. Generalmente, la evolución es benigna durante años pero a veces existe progresión de lesiones nodulares localizadas a amiloidosis sistémica en menos del $15 \%$ de los casos o en ocasiones desarrolla paraproteinemia (5-12).

No existen criterios clínicos para el diagnóstico. La amiloidosis es una entidad que clínicamente se sospecha. Se ha señalado la asociación con macroglosia no geográfica y síndrome del túnel carpiano en la amiloidosis sistémica. En la piel se debe sospechar si hay dermatosis con pápulas y máculas altamente pruriginosas (4).

El procedimiento diagnóstico consiste en la biopsia con aguja fina que incluye grasa subcutánea y la superficie afectada, esta suele ser positiva en el $80 \%$ de los casos de la entidad. Esta prueba es altamente sensible y fue descrita por primera vez en 1973 por Westermark y Stenkvist $(5,6)$. Si la biopsia resulta negativa y existe sospecha de amiloidosis sistémica, se debe realizar biopsia de la mucosa rectal, la cual es positiva en el $70 \%$ de los casos. Si tras estos procedimientos no se llega al diagnóstico se practicará biopsia del órgano presumiblemente afectado.

Deben realizarse estudios generales como las pruebas hematológicas y de química sanguínea. Algunas específicas dependen de la sospecha del órgano que pueda estar afectado. Cuando están alterados en la radiografía de tórax, se encuentra predominio de patrón intersticial o nodular. En el electrocardiograma, el bajo voltaje generalizado es sugerente de amiloidosis, aunque también existen anomalías en la conducción auriculoventricular e interventricular. En el ecocardiograma bidimensional se puede identificar engrosamiento simétrico de las paredes del ventrículo izquierdo y del tabique interventricular con hipocinesia y disminución del tamaño de las cavidades izquierdas. Puede haber disfunción diastólica con patrón restrictivo sin evidencia de enfermedad coronaria y sin afectación valvular. La infiltración miocárdica por amiloide se traduce por una imagen consistente en un granulado brillante (granular sparkling) en la pared del ventrículo izquierdo y septum interventricular (4-7).

Se encuentra componente monoclonal en el $90 \%$ de los casos. Del $5-15 \%$ de los pacien-

\begin{tabular}{|l|l|l|l|}
\hline \multicolumn{4}{|c|}{ CARACTERÍSTICAS CLÍNICAS DE LA AMILOIDOSIS CUTÁNEA } \\
\hline \multicolumn{1}{|c|}{ Variables } & Amiloidosis liquenoide & Amiloidosis macular & Amiloidosis nodular \\
\hline Edad & Variable & 30-60 años & Variable \\
\hline Lesión & $\begin{array}{l}\text { Pápulas lisas- } \\
\text { hiperqueratosis -placas } \\
\text { pluriticas }\end{array}$ & $\begin{array}{l}\text { Lesión delineada, } \\
\text { parcheada, } \\
\text { hiperpigmentada, } \\
\text { prurito }\end{array}$ & $\begin{array}{l}\text { Nódulos cereos - } \\
\text { bullas-prurito-atrofia- } \\
\text { hemorragia cutánea }\end{array}$ \\
\hline Ubicación & $\begin{array}{l}\text { Extremidad inferior y } \\
\text { superior- tronco }\end{array}$ & $\begin{array}{l}\text { Extremidad superior } \\
\text { o inferior-espalda } \\
\text { superior }\end{array}$ & $\begin{array}{l}\text { Extremidad superior e } \\
\text { inferior-genitales-cara }\end{array}$ \\
\hline $\begin{array}{l}\text { Diagnóstico } \\
\text { diferencial }\end{array}$ & Liquen plano & Melanosis- vitiligo & Amiloidosis sistémica \\
\hline
\end{tabular}


tes con mieloma múltiple presentarán asociadamente amiloidosis cutánea (6).

Las recomendaciones sobre el tratamiento son muy variables ya que no existe tratamiento que demuestre mayor eficacia frente a los demás. Solo se deberá tratar a los pacientes sintomáticos. Los tratamientos tópicos incluyen corticosteroides, (betametasona), calcipotriol, tacrolimus $0,1 \%$ y dimetilsulfóxido tópico. La fototerapia se ha intentado. Los tratamientos físicos incluyen dermoabrasión (7). Los depósitos de amiloide macular, liquen amiloide y nodular se pueden extirpar quirúrgicamente o tratar con láser de dióxido de carbono, pero tienden a recurrir. La forma maculosa, papulosa y liquen amiloide en algunos casos responden mal a los esteroides tópicos combinados con antihistamínicos sistémicos, al igual la recurrencia es alta con la crioterapia, curetaje, incluso inyección de corticoide intralesional, y el mismo laser de c02 (8-9). Hay estudios que evalúan la eficacia del uso de ciclofosfamida en la amiloidosis cutánea localizada primaria. $(8,9)$. Se utilizó ciclofosfamida $50 \mathrm{mg}$ / día vía oral y el prurito fue el primer síntoma en desaparecer.

No hay estudios adecuados para ponderar el efecto del tratamiento. El enfoque terapéutico se basa en tres objetivos: [A] reducir la síntesis de la proteína precursora de las fibrillas amiloide, [B] evitar el depósito y polimerización de la misma, [C] tratamiento sintomático de los órganos afectados (10-11).
El pronóstico de las distintas amiloidosis cutáneas es muy variable, depende de dos factores principales: la presencia de fibrillas de amiloide en toda la dermis, incluidas las paredes vasculares y la obtención de la cadena ligera de inmunoglobulina de las fibrillas de amiloide. Estas dos características se observan en la amiloidosis nodular, aunque algunos autores demostraron que la amiloidosis cutánea nodular surge en relación con el plasmocitoma cutáneo (12). Existe controversia acerca de si la enfermedad es una amiloidosis localizada o una forma temprana de la amiloidosis sistémica, por tanto, el seguimiento a largo plazo es importante $(8,9)$.

\section{CONCLUSIÓN}

La amiloidosis cutánea sin compromiso de otros órganos es de buen pronóstico, entidad inflamatoria, crónica, poco frecuente y de usual diagnóstico tardío. Pese a ello, debe realizarse seguimiento a largo plazo.

\section{CONFLICTO DE INTERESES: ninguno que declarar.}

FINANCIACIÓN: recursos propios de los autores. Costos médicos asistenciales, estudios paraclínicos y los inherentes a las biopsias, así como los honorarios profesionales, fueron cubiertos dentro de las actividades asistenciales.

\section{REFERENCIAS BIBLIOGRÁFICAS}

1. Steciuk A, Dompmartin A, Troussard X, Verneuil L, Macro M, Comoz F, Leroy D. Cutaneous amyloidosis and possible association with systemic amyloidosis. International Journal of Dermatology.2002;41(3):127-32.

2. Cortez F, Betanzos A, Corayhua D, Medina J, García S, Leyva M. Amiloidosis cutánea nodular. Dermatol.Perú. 2005;15(2):161-63.

3. Touart D, Purnima S. Cutaneous deposition diseases I. J Am Acad Dermatol. 1998; 39(2):149-71.

4. Palma C, Grunholz D, Osorio G. Características clínicas de pacientes con diagnóstico histopatológico de amiloidosis. Rev Med Chil. 2005;133:655-61.

5. Nguyen NV. Nodular localized primary cutaneous amyloidosis. Clin Exp Dermatol. 1987;12:460-2.

6. Glenner GG. Amyloid deposits and amyloidosis. The beta-fibrilloses. N Engl J Med. 1980;302(23):1283-92.

7. Breathnach SM.Amyloid and amiloidosis. Am J Acad Dermatol 1988; 18:1- 16.

8. Schwendiman, MN.; Beachkofsky, TM.; Wisco, OJ.; Owens, NM.; Hodson, DS.: Primary cutaneous nodular amyloidosis: case report and review of the literature. Cutis 2009; 84: 87-92.

9. Meijer, J.M.; Schonland, S.O.; Palladini, G.; Merlini, G.; Hegenbart, U.; Ciocca, O.; Perfetti, V.; Leijsma, M.K.; Bootsma, H.; Hazenberg, B.P.: Sjögren's Syndrome and Localized Nodular Cutaneous Amyloidosis. Arthritis Rheum 2008; 58: 1992-1999.

10. Pérez-Ríos DP, Méndez-Landín CC, Cruz-Ortíz H, García-Benítez SV, Aguilar-Martínez E, Peñaloza-Ramírez R. Macroglosia nodular por amiloidosis asociada a mieloma múltiple. Reporte de un caso. Rev Med Hosp Gen Mex. 2014; 77(1):43-46.

11. Eder L, Bitterman H. Image in clinical medicine. Amyloid purpura. N Engl J Med 2007; 356:2406.

12. Masuda C, Mohri S. Nakajima. Histopathological and immunohistochemical study of amyloidosis cutis nodularis atrophicans. Comparison with systemic amyloidosis. $\mathrm{Br}$ J Dermatol 1988;119(1): 33-43. 